\title{
Berry-Esséen bounds and almost sure CLT for quadratic variation of weighted fractional Brownian motion
}

\author{
Guangjun Shen ${ }^{1 *}$, Litan Yan ${ }^{2}$ and Jing Cui ${ }^{1}$
}

\author{
"Correspondence: gjshen@163.com \\ 1 Department of Mathematics, \\ Anhui Normal University, 1 East \\ Beijing Rd., Wuhu, 241000, P.R. China \\ Full list of author information is \\ available at the end of the article
}

\begin{abstract}
In this paper, using the recent results on Stein's method combining with Malliavin calculus and the almost sure central limit theorem for sequences of functionals of general Gaussian fields developed by Nourdin and Peccati, we derive the explicit bounds for the Kolmogorov distance in the central limit theorem and obtain the almost sure central limit theorem for the quadratic variation of the weighted fractional Brownian motion.

MSC: $60 \mathrm{~F} 05 ; 60 \mathrm{G} 15 ; 60 \mathrm{H} 07$

Keywords: weighted fractional Brownian motion; almost sure central limit theorem; Malliavin calculus; Stein's method; multiple stochastic integral
\end{abstract}

\section{Introduction}

Self-similar stochastic processes with long range dependence are of practical interest in various applications, including econometrics, Internet traffic and hydrology. These are processes $X=\left\{X_{t}: t \geq 0\right\}$ whose dependence on the time parameter $t$ are self-similar in the sense that there exists a (self-similarity) parameter $H \in(0,1)$ such that for any constant $c \geq 0,\left\{X_{c t}: t \geq 0\right\}$ and $\left\{c^{H} X_{t}: t \geq 0\right\}$ have the same distribution. These processes are often endowed with other distinctive properties.

The fractional Brownian motion (fBm for short) is the usual candidate to model phenomena in which the self-similarity property can be observed from the empirical data. The $\mathrm{fBm}$ is a suitable generalization of the standard Brownian motion, which exhibits long-range dependence, self-similarity and has stationary increments. Some surveys and complete literatures could be found in Biagini et al. [1], Hu [2], Mishura [3], Nualart [4]. On the other hand, many authors have proposed to use more general self-similar Gaussian processes and random fields as stochastic models. Such applications have raised many interesting theoretical questions about self-similar Gaussian processes and fields in general. Therefore, some generalizations of the $\mathrm{fBm}$ such as bi-fractional Brownian motion, sub-fractional Brownian motion and the weighted fractional Brownian motion have been introduced. However, in contrast to the extensive studies on $\mathrm{fBm}$, there has been little systematic investigation on other self-similar Gaussian processes. The main reason for this is the complexity of dependence structures for self-similar Gaussian processes which do not have stationary increments.

o 2013 Shen et al. licensee Springer. This is an Open Access article distributed under the terms of the Creative Commons Attribution License (http://creativecommons.org/licenses/by/2.0), which permits unrestricted use, distribution, and reproduction in any medium, provided the original work is properly cited. 
In this paper, we consider the weighted fractional Brownian motion (wfBm for short). The so-called wfBm $B^{a, b}$ with parameters $a>-1,|b|<1,|b| \leq a+1$ is a centered and self-similar Gaussian process with long/short-range dependence. It admits the relatively simple covariance as follows:

$$
E\left[B_{t}^{a, b} B_{s}^{a, b}\right]=R^{a, b}(t, s):=\int_{0}^{s \wedge t} u^{a}\left[(t-u)^{b}+(s-u)^{b}\right] d u, \quad s, t \geq 0 .
$$

Clearly, for $a=0, b=0, B^{a, b}$ coincides with the standard Brownian motion $B$. For $a=0$, (1.1) reduces to

$$
E\left[B_{t}^{a, b} B_{s}^{a, b}\right]=\frac{1}{b+1}\left[t^{b+1}+s^{b+1}-|s-t|^{b+1}\right],
$$

which for $-1<b<1$ corresponds to the covariance of the well-known $\mathrm{fBm}$ with Hurst parameter $\frac{b+1}{2}$ and it admits the explicit significance. Hence, wfBm's are a family of processes which extend fBm's. This process $B^{a, b}$ appeared in Bojdecki et al. [5] in a limit of occupation time fluctuations of a system of independent particles moving in $\mathbb{R}^{d}$ according to a symmetric $\alpha$-stable Lévy process, $0<\alpha \leq 2$, started from an inhomogeneous Poisson configuration with intensity measure

$$
\frac{d x}{1+|x|^{\gamma}}
$$

and $0<\gamma \leq d=1<\alpha, a=-\gamma / \alpha, b=1-1 / \alpha$, the ranges of values of $a$ and $b$ being $-1<a<0$ and $0<b \leq 1+a$. The process $B^{a, b}$ also appeared in Bojdecki et al. [6] in a high density limit of occupation time fluctuations of the above mentioned particles system, where the initial Poisson configuration has finite intensity measure, with $d=1<\alpha, a=-1 / \alpha, b=1-1 / \alpha$. Moreover, wfBm was first studied by Bojdecki et al. [7], and it is neither a semimartingale nor a Markov process unless $a=0, b=0$, so many powerful techniques from stochastic analysis are not available when dealing with $B^{a, b}$. The wfBm has properties analogous to those of $\mathrm{fBm}$ (self-similarity, long-range dependence, Hölder paths). However, in comparison with $\mathrm{fBm}$, the wfBm has non-stationary increments. On the other hand, Garzón [8] showed that for certain values of the parameters the weighted fractional Brownian sheets were obtained as limits in law of occupation time fluctuations of a stochastic particle model.

Stein's method is a general method in probability theory to obtain bounds on the distance between two probability distributions with respect to a probability metric (see Stein [9]). The Malliavin calculus is an infinite-dimensional differential calculus, involving operators defined on the class of functionals of a given Gaussian stochastic process. Recently, Nourdin and Peccati $[10,11]$ unveiled the surface of the deep connection between the Malliavin calculus and Stein's method in order to derive explicit bounds in the Gaussian and gamma approximations of random variables in a fixed Wiener chaos of a general Gaussian process. In particular, such an approach implies that on any fixed Wiener chaos each one of the well-known distances (Kolmogorov, total variation, Wasserstein) generates the weak topology when the limit is a Gaussian random variable. Some important non-linear functionals of Gaussian processes can be written as multiple stochastic integrals. An important particular example is the quadratic variation which lies in the second 
Wiener chaos. In the case of $\mathrm{fBm}$, Breuer and Major [12] proved the central limit theorem for the quadratic variation when the Hurst parameter $H \in\left(0, \frac{3}{4}\right)$, Breton and Nourdin [13] did it for the critical value $H=\frac{3}{4}$. Park et al. [14] studied a central limit theorem of crossvariation related to the standard Brownian sheet and provided the exact Berry-Esséen bound on the Kolmogorov distance. Kim [15] studied a central limit theorem of the cross variation related to fractional Brownian sheet with Hurst parameter $H=\left(H_{1}, H_{2}\right)$ such that $\frac{1}{4}<H_{1}, H_{2}<\frac{1}{2}$. On the other hand, Bercu et al. [16] studied the almost sure central limit theorems for sequences of functionals of general Gaussian fields. Tudor [17] proved the almost sure central limit theorem for the quadratic variation of the sub-fractional Brownian motion. Aazizi et al. [18] and Liu [19] studied the bi-fractional Brownian motion case, respectively.

Motivated by all these results, in the present work, we consider the explicit bounds for the Kolmogorov distance in the central limit theorem and almost sure central limit theorems (ASCLT in short) for the quadratic variation of wfBm. The above mentioned properties make wfBm a possible candidate for models which involve long-range dependence, self-similarity and non-stationarity. Therefore, it seems interesting to study the BerryEsséen bounds and ASCLT for the quadratic variation of the wfBm.

This paper is organized as follows. Section 2 contains some preliminaries for the Malliavin calculus and main results: Theorem 2.1 and Theorem 2.2. The proofs of the main results are given in Section 3.

We will use $c_{a, b}$ and $C_{a, b}$ to denote positive and finite constants depending on $a, b$ only which may not be the same in each occurrence.

\section{Malliavin calculus on wfBm and main results}

In this section, we present the basic elements of Gaussian analysis and the Malliavin calculus that are used in this paper. Some surveys and a complete list of literature could be found in Nualart [4].

Let $\mathcal{H}$ be a real separable Hilbert space. For any $q \geq 1$, we denote $\mathcal{H}^{\otimes q}$ to be the $q$ th tensor product of $\mathcal{H}$ and $\mathcal{H} \odot q$ to be the associated $q$ th symmetric tensor product. We write $X=\{X(h), h \in \mathcal{H}\}$ to indicate a centered isonormal Gaussian process over $\mathcal{H}$ defined on some probability space $(\Omega, \mathscr{F}, P)$. This means that $X$ is a centered Gaussian family, whose covariance is given in terms of the scalar product of $\mathcal{H}$ by $\mathbb{E}[X(h) X(g)]=\langle h, g\rangle_{\mathcal{H}}$.

For every $q \geq 1$, let $\mathscr{H}_{q}$ be the $q$ th Wiener chaos of $X$, that is, the closed linear subspace of $L^{2}(\Omega, \mathscr{F}, P)$ generated by the family of random variables $\left\{H_{q}(X(h)), h \in \mathcal{H},\|h\|_{\mathcal{H}}=1\right\}$, where $H_{q}$ is the $q$ th Hermite polynomial defined as

$$
H_{q}(x)=(-1)^{q} e^{\frac{x^{2}}{2}} \frac{d^{q}}{d x^{q}}\left(e^{-\frac{x^{2}}{2}}\right)
$$

The mapping $I_{q}\left(h^{\otimes q}\right)=H_{q}(X(h))$ can be extended to a linear isometry between the symmetric tensor product $\mathcal{H}^{\odot q}$ equipped with the modified norm $\|\cdot\|_{\mathcal{H}^{\odot q}}=\sqrt{q !}\|\cdot\|_{\mathcal{H}^{\otimes q}}$ and the $q$ th Wiener chaos $\mathscr{H}_{q}$. Then

$$
\mathbb{E}\left[I_{p}(f) I_{q}(g)\right]=\delta_{p, q} p !\langle f, g\rangle_{\mathcal{H}}^{\otimes p},
$$

where $\delta_{p, q}$ stands for the usual Kronecker symbol, for $f \in \mathcal{H}^{\odot p}, g \in \mathcal{H}^{\odot q}$ and $p, q \geq 1$. Moreover, if $f \in \mathcal{H}^{\otimes q}$, we have $I_{q}(f)=I_{q}(\widetilde{f})$, where $\widetilde{f} \in \mathcal{H}^{\odot q}$ is the symmetrization of $f$. 
Let $\left\{e_{k}, k \geq 1\right\}$ be a complete orthonormal system in $\mathcal{H}$. Given $f \in \mathcal{H}^{\odot p}, g \in \mathcal{H}^{\odot q}$, for every $r=0,1,2, \ldots, p \wedge q$, the contraction of $f$ and $g$ of order $r$ is the element of $\mathcal{H}^{\otimes(p+q-2 r)}$ defined by

$$
f \otimes_{r} g=\sum_{i_{1}, \ldots, i_{r}=1}^{\infty}\left\langle f, e_{i_{1}} \otimes \cdots \otimes e_{i_{r}}\right\rangle_{\mathcal{H}^{\otimes r}} \otimes\left\langle g, e_{i_{1}} \otimes \cdots \otimes e_{i_{r}}\right\rangle_{\mathcal{H}^{\otimes r}}
$$

Since $f \otimes_{r} g$ is not necessarily symmetric, we denote its symmetrization by $f \widetilde{\otimes}_{r} g \in$ $\mathcal{H}^{\odot(p+q-2 r)}$. Note that $f \otimes_{0} g=f \otimes g$ equals the tensor product of $f$ and $g$, for $p=q$, $f \otimes_{q} g=\langle f, g\rangle_{\mathcal{H}}^{\otimes q}$, namely the scalar product of $f$ and $g$. In particular, when $\mathcal{H}=L^{2}(A, \mathcal{A}, \mu)$, where $(A, \mathcal{A})$ is a measurable space and $\mu$ is a $\sigma$-finite and non-atomic measure, one has that $\mathcal{H}^{\otimes q}=L_{S}^{2}\left(A^{q}, \mathcal{A}^{\otimes q}, \mu^{\otimes q}\right)$ is the space of symmetric and square integrable functions on $A^{q}$. In this case, (2.2) can be rewritten as

$$
\begin{aligned}
\left(f \otimes_{r} g\right)\left(t_{1}, \ldots, t_{p+q-2 r}\right)= & \int_{A^{r}} f\left(t_{1}, \ldots, t_{p-r}, s_{1}, \ldots, s_{r}\right) \\
& \times g\left(t_{p-r+1}, \ldots, t_{p+q-2 r}, s_{1}, \ldots, s_{r}\right) d \mu\left(s_{1}\right) \cdots d \mu\left(s_{r}\right),
\end{aligned}
$$

that is, we identify $r$ variables in $f$ and $g$ and integrate them out.

The following product (multiplication) formula is very useful: if $f \in \mathcal{H}^{\otimes p}, g \in \mathcal{H}^{\otimes q}$, then

$$
I_{p}(f) I_{q}(g)=\sum_{r=0}^{p \wedge q} r ! C_{p}^{r} C_{q}^{r} I_{p+q-2 r}\left(f \otimes_{r} g\right) .
$$

Let us now introduce some basic elements of the Malliavin calculus with respect to the isonormal Gaussian process $X$. Let $\mathscr{S}$ be a set of smooth cylindrical functionals of the form

$$
F=f\left(X\left(\varphi_{1}\right), \ldots, X\left(\varphi_{n}\right)\right)
$$

where $n \geq 1$ and $f \in C_{b}^{\infty}\left(\mathbb{R}^{n}\right)$ and $\varphi_{i} \in \mathcal{H}$. The Malliavin derivative of a functional $F$ defined as above is given by

$$
D F=\sum_{i=1}^{n} \frac{\partial f}{\partial x_{i}}\left(X\left(\varphi_{1}\right), \ldots, X\left(\varphi_{n}\right)\right) \varphi_{i}
$$

and this operator can be extended to the closure $\mathbb{D}^{m, 2}(m \geq 1)$ of $\mathscr{S}$ with respect to the norm

$$
\|F\|_{m, 2}^{2} \equiv \mathbb{E}|F|^{2}+\mathbb{E}\|D F\|_{\mathcal{H}}^{2}+\cdots+\mathbb{E}\left\|D^{m} F\right\|_{\mathcal{H}^{\odot m}}^{2},
$$

where $\mathcal{H}^{\odot m}$ denotes the $m$-fold symmetric tensor product of $\mathcal{H}$, and the $m$ th derivative $D^{m}$ is defined by iteration. The Malliavin derivative satisfies the following chain rule. For every random variable $F=\left(F_{1}, F_{2}, \ldots, F_{n}\right)$ with components in $\mathbb{D}^{1,2}$ and for every continuously differentiable function $\psi: \mathbb{R} \rightarrow \mathbb{R}$ with bounded partial derivative, we obtain 
$\psi\left(F_{1}, \ldots, F_{n}\right) \in \mathbb{D}^{1,2}$, and for any $s \in[0,1]$

$$
D_{s} \psi\left(F_{1}, \ldots, F_{n}\right)=\sum_{i=1}^{n} \frac{\partial \psi}{\partial x_{i}}\left(F_{1}, \ldots, F_{n}\right) D_{s} F_{i}
$$

From Bojdecki et al. [7] (see also Yan and An [20]) we have

$$
c_{a, b}(t \vee s)^{a}|t-s|^{b+1} \leq E\left[\left(B_{t}^{a, b}-B_{s}^{a, b}\right)^{2}\right] \leq C_{a, b}(t \vee s)^{a}|t-s|^{b+1}
$$

for $s, t \geq 0$. In particular, we have

$$
E\left[\left(B_{t}^{a, b}-B_{s}^{a, b}\right)^{2}\right] \leq C_{a, b}|t-s|^{a+b+1}
$$

for $a \leq 0$.

Thus, Kolmogorov's continuity criterion implies that wfBm is Hölder continuous of or$\operatorname{der} \delta$ for any $\delta<\frac{1}{2}(1+b)$. We can write its covariance as

$$
R^{a, b}(t, s)=\int_{0}^{t \wedge s} u^{a}(t \vee s-u)^{b} d u+\frac{1}{2}(t \wedge s)^{a+b+1},
$$

which gives

$$
\frac{\partial^{2}}{\partial t \partial s} R^{a, b}(t, s)=b(t \wedge s)^{a}(t \vee s-t \wedge s)^{b-1}
$$

for $b>0$. Clearly, the function $(t, s) \mapsto R^{a, b}(t, s)$ is of class $C^{2}\left((0, T]^{2}\right)$ and it is the distribution function of an absolutely continuous positive measure with density $\frac{\partial^{2}}{\partial s \partial r} R^{a, b}(t, s)$ belonging to $L^{1}\left([0, T]^{2}\right)$ for $b>0$.

Now, we assume that $b>0$. The canonical Hilbert space $\mathcal{H}$ associated to the wfBm is defined as the completion of the linear space $\mathcal{E}$ generated by the indicator functions $\left\{1_{[0, t]}, t \in[0, T]\right\}$ with respect to the inner product

$$
\left\langle 1_{[0, t]}, 1_{[0, s]}\right\rangle_{\mathcal{H}}=R^{a, b}(t, s) .
$$

The application $\mathcal{E} \ni \varphi \mapsto \xi(\varphi)$ is an isometry from $\mathcal{E}$ to the Gaussian space generated by $B^{a, b}$ and it can be extended to $\mathcal{H}$. The Hilbert space $\mathcal{H}$ can be written as

$$
\mathcal{H}=\left\{\varphi:[0, T] \rightarrow \mathbb{R} \mid\|\varphi\|_{\mathcal{H}}<\infty\right\}
$$

where

$$
\|\varphi\|_{\mathcal{H}}^{2}:=\int_{0}^{T} \int_{0}^{T} \varphi(t) \varphi(s) \phi(t, s) d t d s
$$

with $\phi(t, s)=(t \wedge s)^{a}(t \vee s-t \wedge s)^{b-1}$. Notice that the elements of the Hilbert space $\mathcal{H}$ may not be functions but distributions of negative order. 
Recall that if $Y, Z$ are two real-valued random variables, then the Kolmogorov distance between the law of $Y$ and the law of $Z$ is defined by

$$
d_{\mathrm{Kol}}(Y, Z)=\sup _{z \in \mathbb{R}}|P(Y \leq z)-P(Z \leq z)| .
$$

The following result due to Nourdin and Peccati (see Theorem 3.1 and Proposition 3.2 of [10]) is very useful and reduces the problem of the normal approximation of multiple stochastic integrals (more general of random variables which are Malliavin derivable) in the Kolmogorov distance (and other standard metrics) to the estimation of the variance of the Malliavin derivative of the multiple integral (of the random variable, respectively).

Proposition 2.1 Let $N$ be a standard normal Gaussian variable, let $q \geq 2$ be an integer and $f \in \mathcal{H}^{\otimes q}$ with $\mathbb{E}\left(\left|I_{q}(f)\right|\right)^{2}=1$. Then

$$
d_{\mathrm{Kol}}\left(I_{q}(f), N\right) \leq \sqrt{\left(\operatorname{Var}\left(\frac{1}{q}\left\|D\left(I_{q}(f)\right)\right\|_{\mathcal{H}}^{2}\right)\right)} .
$$

Definition 2.1 A sequence $\left(X_{n}\right)_{n \geq 1}$ of random variables satisfies the almost sure central limit theorem (ASCLT for short) if a.s.

$$
\frac{1}{\log n} \sum_{k=1}^{n} \frac{1}{k} \varphi\left(X_{k}\right) \rightarrow \mathbb{E}[\varphi(N)]
$$

as $n$ tends to infinity, for every $\varphi: \mathbb{R} \rightarrow \mathbb{R}$ bounded and continuous function, where $N \sim$ $\mathcal{N}(0,1)$.

The following convenient criterion which extends the convergence in law to almost surely convergence (in particular for ASCLT) is due to Ibragimov and Lifshits [21]. It plays a crucial role in all of the sequel.

Proposition 2.2 Assume that $X_{n}$ converges in law to $X$. If for any $r>0$,

$$
\sup _{|t| \leq r} \sum_{n} \frac{1}{n \log n} \mathbb{E}\left[\frac{1}{\log ^{2} n}\left|\sum_{k=1}^{n} \frac{1}{k}\left(e^{i t X_{k}}-\mathbb{E} e^{i t X}\right)\right|^{2}\right]<\infty
$$

then a.s.

$$
\frac{1}{\log n} \sum_{k=1}^{n} \frac{1}{k} \varphi\left(X_{k}\right) \rightarrow \mathbb{E}[\varphi(X)]
$$

as $n$ tends to infinity, for every $\varphi: \mathbb{R} \rightarrow \mathbb{R}$ bounded and continuous function.

Applying the above criterion to multiple stochastic integrals, Bercu et al. [16] proved the following ASCLT.

Proposition 2.3 Let $X_{n}=I_{q}\left(f_{n}\right), q \geq 2, f_{n} \in \mathcal{H}^{\otimes q}$ be such that $\mathbb{E} X_{n}^{2}=1$ and $X_{n}$ converges weakly to $N \sim \mathcal{N}(0,1)$. If the following conditions are satisfied: 
(1) For every $1 \leq r \leq q-1$,

$$
\sum_{n=2}^{\infty} \frac{1}{n \log ^{2} n} \sum_{k=1}^{n} \frac{1}{k}\left\|f_{k} \otimes_{r} f_{k}\right\|_{\mathcal{H}^{\otimes 2(q-r)}}<\infty
$$

(2)

$$
\sum_{n=2}^{\infty} \frac{1}{n \log ^{3} n} \sum_{k, l=1}^{n} \frac{1}{k l}\left|\mathbb{E}\left(X_{k} X_{l}\right)\right|<\infty
$$

then $\left(X_{n}\right)_{n}$ satisfies the ASCLT.

Let $\left\{B_{t}^{a, b}\right\}_{t \geq 0}$ be a weighted fractional Brownian motion. Define

$$
Z_{n}=\sum_{k=0}^{n-1} n^{1+a+b}\left[\left(B_{(k+1) / n}^{a, b}-B_{k / n}^{a, b}\right)^{2}-\operatorname{Var}\left(B_{(k+1) / n}^{a, b}-B_{k / n}^{a, b}\right)\right]
$$

The aim of the present work is to state and prove the following two results.

Theorem 2.1 Let $N \sim \mathcal{N}(0,1)$. Then $\frac{Z_{n}}{\sqrt{\operatorname{Var}\left(Z_{n}\right)}}$ converges in distribution to $N$ as $n$ tends to infinity and there exists a constant $C_{a, b}$ depending only on $a, b$, such that for every $n \geq 1$ the following Berry-Esséen bounds hold:

$$
d_{\mathrm{Kol}}\left(\frac{Z_{n}}{\sqrt{\operatorname{Var}\left(Z_{n}\right)}}, N\right) \leq C_{a, b} \begin{cases}\frac{1}{\sqrt{n}}, & \frac{1+a+b}{2} \in\left(0, \frac{1}{2}\right), \\ n^{(1+a+b)-\frac{3}{2}}, & \frac{1+a+b}{2} \in\left[\frac{1}{2}, \frac{3}{4}\right), \\ \frac{1}{\sqrt{\log n}}, & \frac{1+a+b}{2}=\frac{3}{4} .\end{cases}
$$

Theorem 2.2 If $\frac{1+a+b}{2} \in\left(0, \frac{3}{4}\right]$, then the sequence $\left(\frac{Z_{n}}{\sqrt{\operatorname{Var}\left(Z_{n}\right)}}\right)_{n \geq 1}$ satisfies the almost sure central limit theorem.

\section{Proof of the main results}

In this section, we assume that $\frac{1+a+b}{2} \in\left(0, \frac{3}{4}\right]$. For simplicity, we denote

$$
\delta_{k / n}=1_{[k / n,(k+1) / n]}
$$

and

$$
\Delta B_{k / n}^{a, b}=B_{(k+1) / n}^{a, b}-B_{k / n}^{a, b}=I\left(\delta_{k / n}\right) .
$$

Proof of Theorem 2.1 By self-similarity property of $B^{a, b}$ (see Bojdecki et al. [7]), we deduce that

$$
\begin{aligned}
n^{1+a+b}\left\langle\delta_{\frac{k}{n}}, \delta_{\frac{l}{n}}\right\rangle_{\mathcal{H}} & =n^{1+a+b} E\left[\left(B_{\frac{k+1}{n}}^{a, b}-B_{\frac{k}{n}}^{a, b}\right)\left(B_{\frac{l+1}{n}}^{a, b}-B_{\frac{l}{n}}^{a, b}\right)\right] \\
& =n^{1+a+b}\left(\frac{1}{n}\right)^{1+a+b} E\left[\left(B_{k+1}^{a, b}-B_{k}^{a, b}\right)\left(B_{l+1}^{a, b}-B_{l}^{a, b}\right)\right] \\
& =\theta(k, l) .
\end{aligned}
$$


Hence, we can write the quadratic variation of $B^{a, b}$, with respect to a subdivision $\pi_{n}=$ $\left\{0<\frac{1}{n}<\frac{2}{n}<\cdots<1\right\}$ of $[0,1]$, as follows:

$$
\begin{aligned}
Z_{n} & =\sum_{k=0}^{n-1} n^{1+a+b}\left[\left(B_{(k+1) / n}^{a, b}-B_{k / n}^{a, b}\right)^{2}-\operatorname{Var}\left(B_{(k+1) / n}^{a, b}-B_{k / n}^{a, b}\right)\right] \\
& =\sum_{k=0}^{n-1}\left[n^{1+a+b}\left(I_{1}\left(\delta_{\frac{k}{n}}\right)\right)^{2}-\theta(k, k)\right] \\
& =I_{2}\left(n^{1+a+b} \sum_{k=0}^{n-1} \delta_{\frac{k}{n}}^{\otimes 2}\right) \\
& :=I_{2}\left(f_{n}\right),
\end{aligned}
$$

where $f_{n}=n^{1+a+b} \sum_{k=0}^{n-1} \delta_{\frac{k}{n}}^{\otimes 2}$. Thus, we can write the correct renormalization of $Z_{n}$ as follows:

$$
V_{n}=\frac{Z_{n}}{\sqrt{\operatorname{Var} Z_{n}}}=\frac{I_{2}\left(f_{n}\right)}{\sqrt{\operatorname{Var} Z_{n}}} .
$$

Therefore, we have

$$
\begin{aligned}
\frac{\operatorname{Var}\left(Z_{n}\right)}{n} & =\frac{1}{n} \mathbb{E}\left[\left|I_{2}\left(f_{n}\right)\right|^{2}\right]=\frac{2}{n}\left\|f_{n}\right\|_{\mathcal{H}^{\otimes 2}}^{2} \\
& =2 n^{2(1+a+b)-1} \sum_{k, l=0}^{n-1}\left\langle\delta_{k / n}^{\otimes 2}, \delta_{l / n}^{\otimes 2}\right\rangle_{\mathcal{H} \otimes 2}=2 n^{2(1+a+b)-1} \sum_{k, l=0}^{n-1}\left\langle\delta_{k / n}, \delta_{l / n}\right\rangle_{\mathcal{H}}^{2} \\
& =\frac{2}{n} \sum_{k=0}^{n-1}\left(n^{(1+a+b)}\left\langle\delta_{k / n}, \delta_{k / n}\right\rangle_{\mathcal{H}}\right)^{2}+4 n^{2(1+a+b)-1} \sum_{0 \leq k<l \leq n-1}\left\langle\delta_{k / n}, \delta_{l / n}\right\rangle_{\mathcal{H}}^{2} \\
& :=2 A_{n}+4 B_{n},
\end{aligned}
$$

where

$$
A_{n}=\frac{1}{n} \sum_{k=0}^{n-1}\left(n^{(1+a+b)}\left\langle\delta_{k / n}, \delta_{k / n}\right\rangle_{\mathcal{H}}\right)^{2}
$$

and

$$
B_{n}=n^{2(1+a+b)-1} \sum_{0 \leq k<l \leq n-1}\left\langle\delta_{k / n}, \delta_{l / n}\right\rangle_{\mathcal{H}}^{2} .
$$

It is clear that $\lim _{n \rightarrow \infty} A_{n}=C_{a, b}$ (by (2.3)). Next, for the term $B_{n}$, we have

$$
\begin{aligned}
B_{n} & =n^{2(1+a+b)-1} \sum_{0 \leq k<l \leq n-1}\left\langle\delta_{k / n}, \delta_{l / n}\right\rangle_{\mathcal{H}}^{2} \\
& =n^{-1} \sum_{0 \leq k<l \leq n-1}\left[n^{1+a+b}\left\langle\delta_{k / n}, \delta_{l / n}\right\rangle_{\mathcal{H}}\right]^{2} \\
& =n^{-1} \sum_{0 \leq k<l \leq n-1}\left[E\left(B_{k+1}^{a, b}-B_{k}^{a, b}\right)\left(B_{l+1}^{a, b}-B_{l}^{a, b}\right)\right]^{2} .
\end{aligned}
$$


Combining (2.3) with Cauchy-Schwarz inequality, it follows that $\lim _{n \rightarrow \infty} B_{n}$ exists and hence

$$
\lim _{n \rightarrow \infty} \frac{\operatorname{Var}\left(Z_{n}\right)}{n} \text { exists. }
$$

According to Proposition 2.1, we need to estimate

$$
C_{n}=\operatorname{Var}\left(\frac{1}{2}\left\|D\left(\frac{Z_{n}}{\sqrt{\operatorname{Var}\left(Z_{n}\right)}}\right)\right\|_{\mathcal{H}}^{2}\right) .
$$

In fact, we have

$$
\begin{aligned}
C_{n} & =\frac{4}{\operatorname{Var}^{2}\left(Z_{n}\right)}\left\|f_{n} \otimes_{1} f_{n}\right\|_{\mathcal{H}^{\otimes 2}}^{2}=\frac{4 n^{2(1+a+b)}}{\operatorname{Var}^{2}\left(Z_{n}\right)}\left\|\sum_{k, l=0}^{n-1} \delta_{k / n}^{\otimes 2} \otimes_{1} \delta_{l / n}^{\otimes 2}\right\|_{\mathcal{H}^{\otimes 2}}^{2} \\
& =\frac{4 n^{2(1+a+b)}}{\operatorname{Var}^{2}\left(Z_{n}\right)}\left\|\sum_{k, l=0}^{n-1}\left\langle\delta_{k / n}, \delta_{l / n}\right\rangle_{\mathcal{H}} \delta_{k / n} \otimes \delta_{l / n}\right\|_{\mathcal{H}^{\otimes 2}}^{2} \\
& =\frac{4 n^{2(1+a+b)}}{\operatorname{Var}^{2}\left(Z_{n}\right)} \sum_{i, j, k, l=0}^{n-1}\left\langle\delta_{k / n}, \delta_{l / n}\right\rangle_{\mathcal{H}}\left\langle\delta_{i / n}, \delta_{j / n}\right\rangle_{\mathcal{H}}\left\langle\delta_{i / n}, \delta_{k / n}\right\rangle_{\mathcal{H}}\left\langle\delta_{j / n}, \delta_{l / n}\right\rangle_{\mathcal{H}} \\
& : \equiv \frac{D(n)}{\operatorname{Var}^{2}\left(Z_{n}\right)} .
\end{aligned}
$$

Then

$$
\begin{aligned}
D(n) \leq & 2 n^{2(1+a+b)} \sum_{i, j, k=0}^{n-1}\left\langle\delta_{i / n}, \delta_{j / n}\right\rangle_{\mathcal{H}}\left\langle\delta_{i / n}, \delta_{k / n}\right\rangle_{\mathcal{H}} \sum_{l=0}^{n-1}\left\langle\delta_{k / n}, \delta_{l / n}\right\rangle_{\mathcal{H}}^{2} \\
& +2 n^{2(1+a+b)} \sum_{i, j, k=0}^{n-1}\left\langle\delta_{i / n}, \delta_{j / n}\right\rangle_{\mathcal{H}}\left\langle\delta_{i / n}, \delta_{k / n}\right\rangle_{\mathcal{H}} \sum_{j=0}^{n-1}\left\langle\delta_{j / n}, \delta_{l / n}\right\rangle_{\mathcal{H}}^{2} \\
:= & 2\left(D_{1}(n)+D_{2}(n)\right) .
\end{aligned}
$$

For the first term $D_{1}(n)$, we obtain

$$
\begin{aligned}
D_{1}(n) \leq & \sum_{i, j, k=0}^{n-1}\left|\left\langle\delta_{i / n}, \delta_{j / n}\right\rangle_{\mathcal{H}}\left\langle\delta_{i / n}, \delta_{k / n}\right\rangle_{\mathcal{H}}\right| \\
& \cdot\left[n^{2(1+a+b)}\left\langle\delta_{k / n}, \delta_{k / n}\right\rangle_{\mathcal{H}}^{2}+2 \sum_{0 \leq k<l \leq n-1} n^{2(1+a+b)}\left\langle\delta_{k / n}, \delta_{l / n}\right\rangle_{\mathcal{H}}^{2}\right] \\
\leq & C_{a, b} \sum_{i, j, k=0}^{n-1}\left|\left\langle\delta_{i / n}, \delta_{j / n}\right\rangle_{\mathcal{H}}\left\langle\delta_{i / n}, \delta_{k / n}\right\rangle_{\mathcal{H}}\right|,
\end{aligned}
$$

since

$$
\left[n^{2(1+a+b)}\left\langle\delta_{k / n}, \delta_{k / n}\right\rangle_{\mathcal{H}}^{2}+2 \sum_{0 \leq k<l \leq n-1} n^{2(1+a+b)}\left\langle\delta_{k / n}, \delta_{l / n}\right\rangle_{\mathcal{H}}^{2}\right]<\infty
$$

if and only if $0<\frac{1+a+b}{2} \leq \frac{3}{4}$. 
We can also get the similar bound for the term $D_{2}(n)$. In particular, with $i=1,2$,

$$
D_{i}(n) \leq C_{a, b} \sum_{i, j, k=0}^{n-1}\left|\left\langle\delta_{i / n}, \delta_{j / n}\right\rangle_{\mathcal{H}}\left\langle\delta_{i / n}, \delta_{k / n}\right\rangle_{\mathcal{H}}\right|
$$

for some positive constant $C_{a, b}$.

The sum on the right-hand side of (3.2) can be estimated in a similar manner as for $D_{i}(n)$ $(i=1,2)$ and this yields

$$
\begin{aligned}
\sum_{i, j, k=0}^{n-1}\left|\left\langle\delta_{i / n}, \delta_{j / n}\right\rangle_{\mathcal{H}}\left\langle\delta_{i / n}, \delta_{k / n}\right\rangle_{\mathcal{H}}\right| \\
\quad=\sum_{i=0}^{n-1}\left[\sum_{j=0}^{n-1}\left|\left\langle\delta_{i / n}, \delta_{j / n}\right\rangle_{\mathcal{H}}\right|\right]^{2} \\
\leq \sum_{i=0}^{n-1}\left[\left\langle\delta_{i / n}, \delta_{i / n}\right\rangle_{\mathcal{H}}+\sum_{j \neq i}^{n-1}\left|\left\langle\delta_{i / n}, \delta_{j / n}\right\rangle_{\mathcal{H}}\right|\right]^{2} \\
\leq \sum_{i=0}^{n-1}\left[C_{a, b} n^{-(1+a+b)}+C_{a, b} \sum_{0 \leq j \leq n-1} j^{(1+a+b)-2}\right]^{2}
\end{aligned}
$$

and therefore

$$
D(n) \leq C_{a, b}\left[n^{1-2(1+a+b)}+n\left(\sum_{j=1}^{n-1} j^{(1+a+b)-2}\right)^{2}\right] .
$$

If $\frac{1+a+b}{2} \in\left(0, \frac{1}{2}\right]$, then from (3.4), the boundedness of $\left(\frac{n^{2}}{\operatorname{Var}^{2}\left(Z_{n}\right)}\right)_{n}$ (by (3.1)) and the convergence of the series $\sum_{j=1}^{n-1} j^{(1+a+b)-2}$, we have

$$
\begin{aligned}
\frac{D(n)}{\operatorname{Var}^{2}\left(Z_{n}\right)} & \leq \frac{C_{a, b}}{\operatorname{Var}^{2}\left(Z_{n}\right)}\left[n^{1-2(1+a+b)}+n\left(\sum_{j=1}^{n-1} j^{(1+a+b)-2}\right)^{2}\right] \\
& \leq C_{a, b} \frac{n^{2}}{\operatorname{Var}^{2}\left(Z_{n}\right)}\left[1+\left(\sum_{j=1}^{n-1} j^{(1+a+b)-2}\right)^{2}\right] n^{-1} \sim O\left(\frac{1}{n}\right) .
\end{aligned}
$$

Assume now $\frac{1}{2}<\frac{1+a+b}{2}<\frac{3}{4}$. Since in this case

$$
\sum_{j=1}^{n-1} j^{(1+a+b)-2} \sim O\left(n^{(1+a+b)-1}\right)
$$

and $\left(\frac{n^{2}}{\operatorname{Var}^{2}\left(Z_{n}\right)}\right)_{n}$ is bounded, we obtain

$$
C_{n} \leq C_{a, b} \frac{n^{2}}{\operatorname{Var}^{2}\left(Z_{n}\right)}\left[n^{-1-2(1+a+b)}+\frac{1}{n}\left(\sum_{j=1}^{n-1} j^{(1+a+b)-2}\right)^{2}\right] \sim O\left(n^{2(1+a+b)-3}\right) .
$$


Assume now that $\frac{1+a+b}{2}=\frac{3}{4}$. Define

$$
\begin{aligned}
U_{n} & =n^{2(1+a+b)-1} \frac{\sum_{1 \leq k<l \leq n-1}\left\langle\delta_{k / n}, \delta_{l / n}\right\rangle_{\mathcal{H}}^{2}}{\log n} \\
& =\frac{\sum_{1 \leq k<l \leq n-1}\left(E\left(B_{k+1}^{a, b}-B_{k}^{a, b}\right)\left(B_{l+1}^{a, b}-B_{l}^{a, b}\right)\right)^{2}}{n \log n} .
\end{aligned}
$$

A straightforward calculation shows that $\lim _{n \rightarrow \infty} U_{n}$ exists. Thus we get that, for $\frac{1+a+b}{2}=\frac{3}{4}$, the estimate

$$
C_{n} \leq C_{a, b} \frac{1}{\log n}
$$

holds. Then we finish the proof of this theorem by Proposition 2.1.

Remark 3.1 The speed of convergence in Theorem 2.1 is the same as for the case of fractional Brownian motion (see Nourdin et al. [11]) and sub-fractional Brownian motion (see Tudor [17]).

To present a proof of Theorem 2.2, the following proposition is needed.

Proposition 3.1 If $a \geq 0$, then the sequence $\left(n^{-\frac{1+a+b}{2}} B_{n}^{a, b}\right)_{n \geq 0}$ satisfies the almost sure central limit theorem.

Proof The proof is straightforward by applying Theorem 4.1 and Corollary 3.7 in Bercu et al. [16] and the fact that

$$
\begin{aligned}
\left|E B_{j}^{a, b} B_{l}^{a, b}\right| & =\int_{0}^{j \wedge l} u^{a}\left[(j-u)^{b}+(l-u)^{b}\right] d u \\
& \leq(j \wedge l)^{a} \int_{0}^{j \wedge l}\left[(j-u)^{b}+(l-u)^{b}\right] d u \\
& =(j \wedge l)^{a} \frac{1}{1+b}\left(j^{b+1}+l^{b+1}-|j-l|^{b+1}\right) \\
& =C_{a, b}\left|E B_{j}^{\frac{b+1}{2}} B_{l}^{\frac{b+1}{2}}\right|,
\end{aligned}
$$

where $B^{\frac{b+1}{2}}$ is a fractional Brownian motion with Hurst index $\frac{b+1}{2}$.

Now we can give the proof of Theorem 2.2.

Proof of Theorem 2.2 From Theorem 2.1, we know that $\left(\frac{Z_{n}}{\operatorname{Var}\left(Z_{n}\right)}\right)_{n}$ satisfies the central limit theorem. Hence we only need to check the conditions (1) and (2) in Proposition 2.3. The rest of the proof could be proved along the line of the proof of Theorem 5.1 of Bercu et al. [16] (see also Theorem 4.2 of Tudor [17], Theorem 4.2 of Aazizi et al. [18], Theorem 2.2 of Liu [19]). For the sake of completeness, we give the main arguments of the proof.

We consider $\frac{1+a+b}{2} \in\left(0, \frac{3}{4}\right)$ and $\frac{1+a+b}{2}=\frac{3}{4}$ separately. 
Case I: Assume that $\frac{1+a+b}{2} \in\left(0, \frac{3}{4}\right)$, by (3.5), (3.6), we obtain

$$
\left\|f_{k} \otimes_{1} f_{k}\right\|_{\mathcal{H}^{\otimes 2}}^{2} \unrhd\left(\frac{1}{n}+n^{2(1+a+b)-3}\right) \unrhd \begin{cases}\frac{1}{n}, & \frac{1+a+b}{2} \in\left(0, \frac{1}{2}\right], \\ n^{2(1+a+b)-3}, & \frac{1+a+b}{2} \in\left(\frac{1}{2}, \frac{3}{4}\right),\end{cases}
$$

where the notation $a_{n} \unrhd b_{n}$ denotes $\sup _{n \geq 1} \frac{\left|a_{n}\right|}{\left|b_{n}\right|}<\infty$. Hence,

$$
\sum_{n=2}^{\infty} \frac{1}{n \log ^{2} n} \sum_{k=1}^{n} \frac{1}{k}\left\|f_{k} \otimes_{1} f_{k}\right\|_{\mathfrak{H}^{\otimes 2}}<\infty
$$

Thus, the condition (1) in Proposition 2.3 is satisfied.

An elementary calculus can show that

$$
\begin{aligned}
& \left\langle f_{k}, f_{l}\right\rangle_{\mathcal{H}^{\otimes 2}} \\
& \quad=\frac{n^{2(1+a+b)}}{\sqrt{\operatorname{Var} Z_{k}} \sqrt{\operatorname{Var} Z_{l}}} \sum_{i=0}^{k-1} \sum_{j=0}^{l-1}\left\langle\delta_{i / n}, \delta_{j / n}\right\rangle_{\mathcal{H}}^{2} \\
& \leq C_{a, b} \frac{n^{2(1+a+b)}}{\sqrt{k l}}\left[\sum_{i=0}^{k-1}\left\langle\delta_{i / n}, \delta_{i / n}\right\rangle_{\mathcal{H}}^{2}+\sum_{i, j=0, i<j}^{k-1}\left\langle\delta_{i / n}, \delta_{j / n}\right\rangle_{\mathcal{H}}^{2}+\sum_{i=0}^{k-1} \sum_{j=k}^{l-1}\left\langle\delta_{i / n}, \delta_{j / n}\right\rangle_{c}^{2}\right] \\
& \leq C_{a, b}\left[\sqrt{\frac{k}{l}}+\frac{1}{\sqrt{k l}} \sum_{i=0}^{k-1}(k-i)^{2(1+a+b)-3}\right] \\
& \quad \leq C_{a, b}\left[\sqrt{\frac{k}{l}}+\frac{1}{\sqrt{k l}} k^{2(1+a+b)-2}\right] \leq C_{a, b} \sqrt{\frac{k}{l}} .
\end{aligned}
$$

It follows from (3.9) that the condition (2) in Proposition 2.3 is satisfied.

Now assume that $\frac{1+a+b}{2}=\frac{3}{4}$. From (3.5) and (3.6) the condition (1) follows easily. We can show that in this case

$$
\begin{aligned}
& \left\langle f_{k}, f_{l}\right\rangle_{\mathcal{H}} \otimes 2 \\
& \leq C_{a, b} \sqrt{\frac{1}{k \log k}} \sqrt{\frac{1}{l \log l}} \sum_{i=0}^{k-1} \sum_{j=0}^{l-1} n^{3}\left\langle\delta_{i / n}, \delta_{j / n}\right\rangle_{\mathcal{H}}^{2} \\
& \leq C_{a, b} \sqrt{\frac{1}{k \log k}} \sqrt{\frac{1}{l \log l}}\left(k+\sum_{i=0}^{k-1} \log (l-i)\right) \\
& \leq C_{a, b} \sqrt{\frac{k \log l}{l \log k}}, \quad \forall k>l .
\end{aligned}
$$

It follows that the condition (2) in Proposition 2.3 is satisfied.

The authors declare that they have no competing interests. 


\section{Author details}

'Department of Mathematics, Anhui Normal University, 1 East Beijing Rd., Wuhu, 241000, P.R. China. ${ }^{2}$ Department of Mathematics, Donghua University, 2999 North Renmin Rd., Songjiang, Shanghai 201620, P.R. China.

\section{Acknowledgements}

The authors would like to thank anonymous earnest referee whose remarks and suggestions greatly improved the presentation of the paper. Guangjun Shen is partially supported by the National Natural Science Foundation of China (11271020), the Natural Science Foundation of Anhui Province (1208085MA11) and the Key Natural Science Foundation of Anhui Educational Committee (KJ2011A139, KJ2012ZD01). Litan Yan is partially supported by the National Natural Science Foundation of China (11171062), Innovation Program of Shanghai Municipal Education Commission (12ZZ063). Jing Cui is partially supported by the Natural Science Foundation of Anhui Province (1308085QA14), the Key Natural Science Foundation of Anhui Educational Committee (KJ2013A133) and the Philosophy and Social Science Planning Foundation of Anhui Province (AHSK11-12D128).

Received: 24 October 2012 Accepted: 17 May 2013 Published: 3 June 2013

\section{References}

1. Biagini, F, Hu, Y, Øksendal, B, Zhang, T: Stochastic Calculus for Fractional Brownian Motion and Applications. Probability and Its Applications. Springer, Berlin (2008)

2. $\mathrm{Hu}, \mathrm{Y}$ : Integral transformations and anticipative calculus for fractional Brownian motions. Mem. Am. Math. Soc. 175, $825(2005)$

3. Mishura, Y: Stochastic Calculus for Fractional Brownian Motion and Related Processes. Lect Notes in Math., vol. 1929. Springer, Berlin (2008)

4. Nualart, D: Malliavin Calculus and Related Topics. Springer, New York (2006)

5. Bojdecki, T, Gorostiza, LG, Talarczyk, A: Occupation time limits of inhomogeneous Poisson systems of independent particles. Stoch. Process. Appl. 118, 28-52 (2008)

6. Bojdecki, T, Gorostiza, LG, Talarczyk, A: Self-similar stable processes arising from high density limits of occupation times of particle systems. Potential Anal. 28, 71-103 (2008)

7. Bojdecki, T, Gorostiza, LG, Talarczyk, A: Some extensions of fractional Brownian motion and sub-fractional Brownian motion related to particle system. Electron. Commun. Probab. 12, 161-172 (2007)

8. Garzón, J: Convergence to weighted fractional Brownian sheets. Commun. Stoch. Anal. 3, 1-14 (2009)

9. Stein, C: A bound for the error in the normal approximation to the distribution of a sum of dependent random variables. In: Proceedings of the Sixth Berkeley Symposium on Mathematical Statistics and Probability, vol. II: Probability Theory, pp. 583-602. University of California Press, Berkeley (1972)

10. Nourdin, I, Peccati, G: Stein's method on Wiener chaos. Probab. Theory Relat. Fields 145, 75-118 (2009)

11. Nourdin, I, Peccati, G: Stein's method meets Malliavin calculus: a short survey with new estimates. In: Duan, J, Luo, S, Wang, C (eds.) Recent Development in Stochastic Dynamics and Stochastic Analysis. Word Scientific, Hackensack (2010)

12. Breuer, P, Major, P: Central limit theorems for nonlinear functionals of Gaussian fields. J. Multivar. Anal. 13, 425-441 (1983)

13. Breton, JC, Nourdin, I: Error bounds for non-normal approximation of Hermite power variations of fractional Brownian motion. Electron. Commun. Probab. 13, 482-493 (2008)

14. Park, HS, Jeon, JW, Kim, YT: The central limit theorem for cross-variation related to the standard Brownian sheet and Berry-Essen bounds. J. Korean Stat. Soc. 40, 239-244 (2011)

15. Kim, YT: Central limit theorem of the cross variation related to fractional Brownian sheet. Commun. Korean Stat. Soc 18, 851-857 (2011)

16. Bercu, B, Nourdin, I, Taqqu, M: Almost sure central limit theorem on the Wiener space. Stoch. Process. Appl. 120 1607-1628 (2010)

17. Tudor, C: Berry-Esséen bounds and almost sure CLT for the quadratic variation of the sub-fractional Brownian motion. J. Math. Anal. Appl. 375, 667-676 (2011)

18. Aazizi, S, Es-Sebaiy, K: Berry-Esséen bounds and almost sure CLT for the quadratic variation of the bifractional Brownian motion. arXiv:1203.2786v3

19. Liu, J: Berry-Esséen bounds and almost sure CLT for quadratic variation of bifractional Brownian motion (submitted)

20. Yan, L, An, L: Itô's formula for weighted fractional Brownian motion. Preprint

21. Ibragimov, IA, Lifshits, MA: On the convergence of generalized moments in almost sure central limit theorem. Theory Probab. Appl. 44(2), 254-272 (2000) 